\title{
MMP-9 inhibition suppresses wear debris-induced inflammatory osteolysis through downregulation of RANK/RANKL in a murine osteolysis model
}

\author{
DESHENG CHEN ${ }^{1,2}$, XIANLONG ZHANG ${ }^{1}$, YONGYUAN GUO ${ }^{1}$,

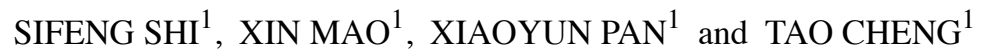 \\ ${ }^{1}$ Department of Orthopaedics, The Sixth Affiliated People's Hospital, Shanghai Jiaotong University \\ School of Medicine, Shanghai 200233; ${ }^{2}$ Department of Orthopaedics, The General Hospital \\ of Ningxia Medical University, Yinchuan 750004, P.R. China
}

Received July 6, 2012; Accepted September 7, 2012

DOI: $10.3892 / \mathrm{ijmm} .2012 .1145$

\begin{abstract}
Wear debris-induced osteolysis in periprosthetic tissue with aseptic loosening is a serious problem after total joint arthroplasty. Matrix metalloproteinase-9 (MMP-9) is expressed in osteoclast cells that surround loosening peri-implant tissue, but the molecular mechanism of MMP-9 action in wear debris-induced osteolysis remains ambiguous. We used a murine osteolysis model to examine the hypothesis that administration of an MMP-9 inhibitor reduces the expression of receptor activator of nuclear factor- $\mathrm{KB}$ (RANK) and nuclear factor- $\kappa \mathrm{B}$ ligand (RANKL) and, thereby, suppressesdebris-induced inflammatory osteolysis. Experiments were performed in 3 groups of 15 mice: a control, a titanium (Ti) and a Ti plus tetracycline group. To provoke inflammatory osteolysis, calvarial bone was implanted from syngeneic littermates, followed by injection of Ti particles into established air pouches for all groups except the control. Tetracycline was administered daily by intraperitoneal (i.p.) injection, and PBS was administered by i.p. injection to the control and Ti groups. Mice were sacrificed 14 days after bone-Ti implantation. Pouch membranes with the intact bone implants were collected for histological and molecular analysis. Tetracycline had minimum effect on the expression of MMP-9 and tumor necrosis factor- $\alpha$ (TNF- $\alpha$ ) but it decreased gene activation and inhibited the expression of RANK and RANKL, thereby inhibiting Ti-particle-induced inflammatory osteolysis. Tetracycline decreased the number of tartrate-resistant acid phosphatase (TRAP)-positive cells in the pouch tissues. Our results in the murine osteolysis model suggest that through the
\end{abstract}

Correspondence to: Dr Xianlong Zhang, Department of Orthopaedics, The Sixth Affiliated People's Hospital, Shanghai Jiaotong University School of Medicine, Shanghai 200233, P.R. China E-mail: zx16412@163.com

Key words: wear debris, titanium particles, matrix metalloproteinase-9, tetracycline, receptor activator of nuclear factor- $\mathrm{\kappa B}$, receptor activator of nuclear factor- $\mathrm{kB}$ ligand downregulation of RANK/RANKL, tetracycline significantly inhibits debris-induced inflammatory osteolysis. Its use in clinical practice may help prevent complications experienced by patients who have undergone total joint arthroplasty.

\section{Introduction}

Total joint arthroplasty is a cost-effective operative procedure for treating dysfunctional weight bearing joints, and relieves pain and restores joint function (1). However, aseptic loosening secondary to wear particle-associated osteolysis is an important cause of complication following hip and knee replacement surgery. Furthermore, revision surgery is associated with significant cost and potential morbidity $(2,3)$.

Titanium (Ti) alloy particles are common wear debris particles in periprosthetic tissue. They stimulate the release and phagocytic activity of monocytes and macrophages around periprosthetic tissue and generate the release of a host of proinflammatory cytokines, including tumor necrosis factor- $\alpha$ (TNF- $\alpha$ ), metalloproteinases (MMPs), interleukin-1 $\beta$ (IL-1 $\beta$ ), interleukin-6 (IL-6), transforming growth factor- $\beta$ (TGF- $\beta$ ), colony stimulating factor-1 (CSF-1) and macrophage-CSF (M-CSF) (4). The resulting inflammatory reaction is widely considered to play a key role in the formation of osteolysis induced by wear debris and finally results in aseptic loosening of the replaced joint (4).

The presence of wear particles from prosthetic implants may also trigger a histiocytic response at the synovial-like membrane which is present at the interface between the bone and the implant. This involves the activation and release of macrophages, fibroblasts, potential proinflammatory cytokines and MMPs, all of which promote bone absorption and osteolysis in periprosthetic tissue (5).

MMPs are a family of proteolytic enzymes that have been reported to be involved in the degradation of the extracellular matrix of various tissues such as bone and have been shown to induce bone resorption (6). MMP-1, -2, -3, -9, -10, -12 and -13 and membrane type 1 (MT-1)-MMP have all been implicated in osteolysis associated with aseptic loosening of joint prostheses (7-10). Matrix metalloproteinase-9 (MMP-9) 
is regarded as a gelatinase $\mathrm{B}$, type IV collagenase and is highly expressed not only during the early stages of osteoclast development, but also in mature osteoclasts which resorb bone (11). Previous studies have demonstrated that MMP-9 activity has an important function in the pathogenesis of dental pulp inflammation (12). A high expression of MMP-9 has also been discovered in human osteoclastomas and osteoclasts in Paget's disease (13). Activation of MMP-9 activity has been demonstrated to be restricted to macrophages in the pseudomembrane of the bone-implant interface (14). Therefore, it constitutes a potential drug target in this indication, and several studies have demonstrated that the inhibition of MMP-9 resulted in a reduced bone resorption in response to wear particles $(15,16)$.

Other studies have demonstrated that the receptor activator of nuclear factor- $\kappa \mathrm{B}$ ligand (RANKL) and the receptor activator of nuclear factor- $\kappa \mathrm{B}$ (RANK) play an important role in regulating wear debris-induced osteoclastogenesis $(17,18)$. Both TNF- $\alpha$ and IL- $1 \beta$ have been demonstrated to be regulated by the expression and activity of the RANK/RANKL in histiocytic tissue around loosened implants $(19,20)$. However, the possible interaction between MMP-9 and RANK/RANKL signaling in the development of inflammatory osteolysis is not well characterized.

The present study using a murine osteolysis model was undertaken to examine the hypothesis that amelioration of $\mathrm{Ti}$ particle-induced inflammatory osteolysis by MMP-9 inhibition is mediated by the downregulation of RANK/RANKL, MMP-9 and TNF- $\alpha$.

\section{Materials and methods}

Titanium alloy particles. Ti particles (diameter 0.1-20 $\mu \mathrm{m}$ ) were obtained from Alfa Aesar Company (Fig. 1). The particles were washed 3 times in $70 \%$ ethanol solution to remove bound endotoxin and were sterilized in $10 \mathrm{mM}$ phosphate-buffered saline (PBS) at $\mathrm{pH}$ 7.4. They were then suspended in sterile $10 \mathrm{mM}$ PBS at a concentration of $15 \mathrm{mg} / \mathrm{ml}$ and stored at $4^{\circ} \mathrm{C}$ until needed. The suspension contained 5\% (weight/volume) particles.

Murine osteolysis model. Female BALB/c mice, aged 8-10 weeks were purchased from the Shanghai Laboratory Animal Centre (Shanghai, China). The mice were randomly assigned to 3 experimental groups with 15 animals in each group: a control group (A), a Ti group (B) and a Ti plus tetracycline group (C).

All mice weighed 20-25 g at the start of the experiment. Previously described techniques were used to establish air pouches (21). Briefly, the dorsal area of each mouse $(2 \times 2 \mathrm{~cm})$ was shaved and sterilized and $2 \mathrm{ml}$ of sterile air was injected subcutaneously to establish an air pouch. To maintain the pouch, $0.5 \mathrm{ml}$ of sterile air was injected into the pouch each day for 6 days.

After 6 days, mice with established air pouches were anaesthetized by i.p. injection of pentobarbital $(50 \mathrm{mg} / \mathrm{kg})$. A $0.5-\mathrm{cm}$ incision overlying the pouch was performed and a section of calvarial bone $(\sim 0.4 \times 0.25 \mathrm{~cm})$ from a genetically identical donor mouse was inserted into the pouch. The $\mathrm{Ti}$ alloy particle suspension $(0.3 \mathrm{ml})$ was injected into estab-

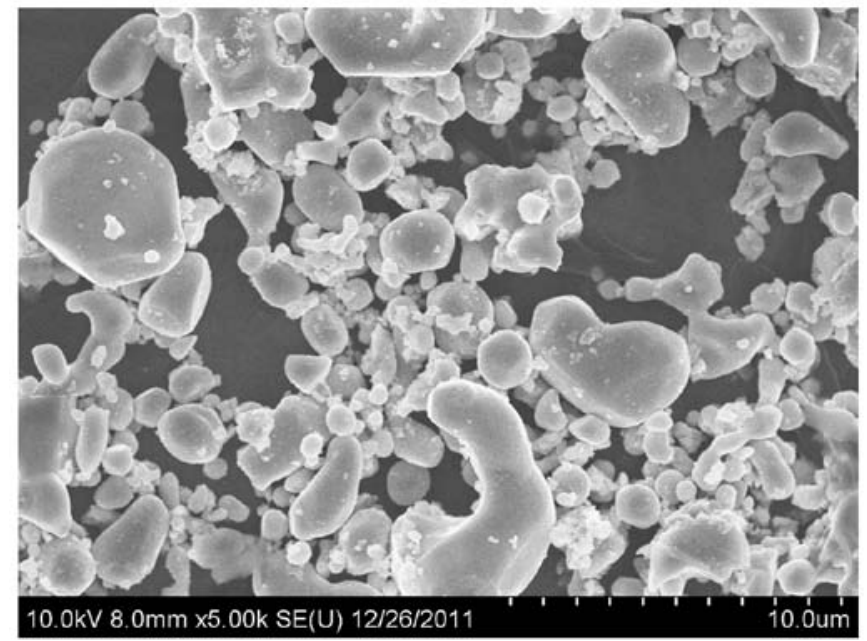

Figure 1. Scanning electron microscopy (SEM) appearance of the Ti particles (magnification, x6,000).

lished air pouches of the mice (except those from group A) to provoke inflammatory osteolysis. The pouch layers and the skin incision were then closed using 4-0 prolene sutures. Tetracycline $50 \mathrm{mg} / \mathrm{kg}$ was administered by i.p. injection to group $\mathrm{C}$ and $0.1 \mathrm{ml}$ sterile PBS was administered by i.p. injection to groups A and B. Injections continued daily until the mice were sacrificed 14 days after bone-Ti implantation. Pouch membranes with the intact bone implants were harvested for histological and molecular analysis.

The Institutional Animal Care and Use Committee of the Shanghai Jiaotong University approved all animal procedures.

Histological evaluation. Tissue samples were fixed in $4 \%$ polyoxymethylene ( $\mathrm{pH} 7.4$ ) for $24 \mathrm{~h}$, after decalcification in $10 \%$ ethylenediaminetetraacetic acid (EDTA). The specimens were processed for dehydration in graded alcohol, cleared in dimethyl benzene and embedded in paraffin. Tissue sections $(6 \mu \mathrm{m})$ were stained with hematoxylin and eosin (H\&E) to evaluate pouch membrane inflammation and implant bone erosion. The stained sections were examined under a light microscope (Olympus DP70; Olympus Optical Co., Tokyo, Japan) and digital photomicrographs were captured and analyzed using a computerized image analysis system with Image-Pro Plus software, version 6.0 (Media Cybernetics, Silver Spring, MD, USA). Pouch membrane thickness and the total numbers of infiltrated cells were measured using digital image analysis.

Four separate sections/specimen were analyzed in a blinded fashion. Pouch membrane thickness was measured at 6 points on each section and 6 random $100-\mu$ m longitudinal pouch areas were selected to count total cells (cells $/ \mathrm{mm}^{2}$ ) based upon nucleus counts.

Expression of tartrate-resistant acid phosphatase (TRAP). TRAP staining was used to identify the presence of osteoclast-like cells in the pouch tissue. Histochemical TRAP staining using a commercial kit (Sigma, St. Louis, MO, USA) was performed on pouches implanted with calvarium. Frozen sections $(6 \mu \mathrm{m})$ were prepared and incubated in xylene for $30 \mathrm{sec}$ and washed with ethanol. Sections were incubated at 
Table I. Gene-specific primers for MMP-9, TNF- $\alpha$, RANK, RANKL and GAPDH.

\begin{tabular}{llll}
\hline Genes & \multicolumn{1}{c}{ Reverse primer sequence } & Forward primer sequence & Size (base pair) \\
\hline MMP-9 & 5'-TACTGGAAGATGTCGTGTGAG-3' & 5'-GGCGTGTCTGGAGATTCG-3' & 112 \\
TNF- $\alpha$ & 5'-CAGGTCACTGTGTCCCAGCATCT-3' & 5'-GAGTCCGGGCAGGTCTACTTT-3' & 235 \\
RANK & 5'-GCGAGGTCTGGCTGACATAC-3' & 5'-CTGCCTCTGGGAACGTGACT-3' & 108 \\
RANKL & 5'-CTGCGTTTTCATGGAGTCTCA-3' & 5'-CAGCATCGCTCTGGTTCCTGTA-3' & 107 \\
GAPDH & 5'-TGCTGTTGAAGTCGCAGGAC-3' & 5'-CCAATGTGTCCGTCGTGGAT-3' & 153 \\
\hline
\end{tabular}

MMP-9, matrix metalloproteinase-9; TNF- $\alpha$, tumor necrosis factor- $\alpha$; RANK, receptor activator of nuclear factor- $\kappa \mathrm{B}$; RANKL, receptor activator of nuclear factor- $\kappa \mathrm{B}$ ligand; GAPDH, glyceraldehyde-3-phosphate dehydrogenase.

$37^{\circ} \mathrm{C}$ for $1 \mathrm{~h}$ in $100 \mathrm{mM}$ acetate buffer ( $\mathrm{pH}$ 5.2) containing $0.5 \mathrm{mM}$ naphthol AS-BI phosphoric acid, $2.2 \mathrm{mM}$ Fast Garnet GBC and $8 \mathrm{mM}$ sodium tartrate. The sections were then washed in several changes of distilled water, followed by counterstaining with a hematoxylin solution. The presence of dark purple staining granules in the cytoplasm was determined as the specific criterion for TRAP-positive cells. Positive TRAP localization was quantified by pixel area count and reported as the percentage of the total implanted bone area in the pouch tissue.

Immunohistochemical staining for MMP-9, TNF- $\alpha, R A N K$ and RANKL. Tissue sections were deparaffinized in xylene and rehydrated in ethanol. The rinsed sections were immersed in antigen retrieval buffer of EDTA in a water bath at $97^{\circ} \mathrm{C}$ for $30 \mathrm{~min}$. The sections were allowed to cool to room temperature before preincubation for $10 \mathrm{~min}$ with $0.3 \%$ (vol/vol) hydrogen peroxide in methanol to suppress the activity of endogenous peroxidase. The sections were blocked with $1.5 \%$ normal goat serum for $30 \mathrm{~min}$. They were then incubated at $4^{\circ} \mathrm{C}$ overnight with the primary antibodies: rabbit anti-MMP-9 (Abcam, USA), mouse anti-TNF- $\alpha$, mouse anti-RANK- $\alpha$ and mouse anti-RANKL- $\alpha$. The sections were rinsed and incubated for $30 \mathrm{~min}$ with biotin-conjugated secondary antibody (Sigma) at $37^{\circ} \mathrm{C}$. Streptavidin-horseradish peroxidase conjugate (SA-HRP) was applied to sections for $30 \mathrm{~min}$ at $37^{\circ} \mathrm{C}$. 3,3'-Diaminobenzidine tetrahydrochloride (DAB) was added for color development with counterstaining using hematoxylin. The digital photomicrographs were analyzed using the Image-Pro image analysis software package.

Gene expression of MMP-9, TNF- $\alpha, R A N K$ and RANKL. Total RNA was extracted from pouch tissue using TRIzol reagent (Invitrogen Life Technologies, Carlsbad, CA, USA) according to the manufacturer's instructions. cDNA was synthesized from the total RNA using a SYBR PrimeScript ${ }^{\mathrm{TM}}$ reverse transcription-polymerase chain reaction (RT-PCR) kit (Takara Bio, Inc., Shiga, Japan). Quantitative real-time RT-PCR was performed to evaluate the relative levels of mRNA for MMP-9, TNF- $\alpha$, RANK and RANKL, using the SYBR PrimeScript ${ }^{\mathrm{TM}}$ RT-PCR kit and an ABI 7500 real-time thermal cycler (Applied Biosystems, Carlsbad, CA, USA) according to the manufacturer's instructions. Gene specific primers for MMP-9, TNF- $\alpha$, RANK and RANKL were designed using Primer 5.0 software (Premier Biosoft International, Palo Alto, CA, USA). The housekeeping gene, glyceraldehyde-3-phosphate dehydrogenase (GAPDH) was used as an internal control. The cycling program involved preliminary denaturation at $95^{\circ} \mathrm{C}$ for $2 \mathrm{~min}$, followed by 40 cycles of denaturation at $95^{\circ} \mathrm{C}$ for $15 \mathrm{sec}$ and annealing at $60^{\circ} \mathrm{C}$ for $1 \mathrm{~min}$.

A comparative threshold cycle $(\mathrm{Ct})$ method with arithmetic formulae was used to determine the relative level of gene expression following PCR amplification. Subtracting the $\mathrm{Ct}$ of the housekeeping gene from the $\mathrm{Ct}$ of the target gene yielded the $\Delta \mathrm{Ct}$ for each group (control and experimental groups) which was entered into the equation $2^{-\Delta \mathrm{Ct}}$. The gene activity in the control group was arbitrarily assigned as 1 to serve as a reference. The primers used in this study were as shown in Table I.

Statistical analysis. Statistical analyses were carried out using the SPSS statistical package, version 11.0 (SPSS, Inc., Chicago, IL, USA). Values are expressed as mean \pm standard deviation (SD). Differences between groups were analyzed using analysis of variance. Values of $\mathrm{P}<0.05$ were considered statistically significant.

\section{Results}

Morphological appearance of $H \& E$-stained sections. Air pouch thickness and cellular infiltration were assessed on H\&E-stained sections (22). Biomaterial particles stimulate tissue inflammation, which is characterized by increased cellular infiltration and pouch membrane thickness (23). As shown in Fig. 2, membrane thickness and frequency of infiltrated cells were significantly increased in the Ti group compared with the control group $(\mathrm{P}<0.05)$. These effects were significantly alleviated by injection with tetracycline $(\mathrm{P}<0.05)$. Bone morphology remained intact in the control group (Fig. 2a), However, bone erosion was observed in pouches stimulated with Ti particles, particularly at the bone-membrane interface where erosion pits were observed (Fig. 2b). Exposure to tetracycline reduced the inflammatory osteolysis response in this murine osteolysis model (Fig. 2c).

Effect of tetracycline on MMP-9 expression. Staining for MMP-9 in the Ti plus tetracycline group was more intense compared to the control group, but less intense compared to the Ti group (Fig. 3a-c). Staining for MMP-9 was predominantly observed in the inflammatory cells surrounding the Ti particles. 


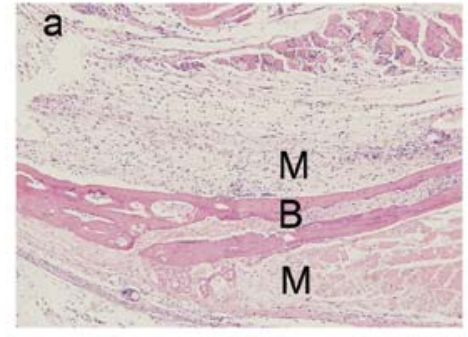

control

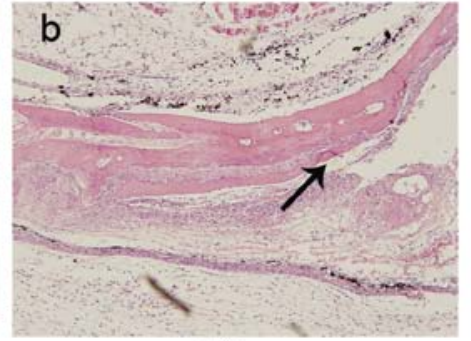

$\mathrm{Ti}$

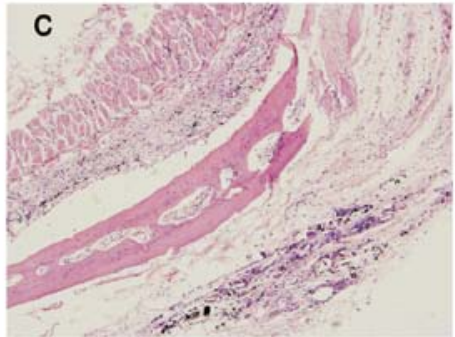

$\mathrm{Ti}+$ Tetracycline

Figure 2. Histological appearance of air pouches after staining with hematoxylin and eosin (H\&E). Ti particles increased the thickness and degree of cellular infiltration into air pouches while tetracycline decreased this response. (a) Air pouch in the control group; (b) air pouch infiltrated with Ti particles; (c) air pouch infiltrated with Ti and treated with tetracycline (magnification, x100). M, membrane; B, bone. The arrow indicates that bone erosion increased remarkably in the TI-particle stimulated bone-implanted pouches, especially in the bone-membrane interface region where erosion pit were observed.

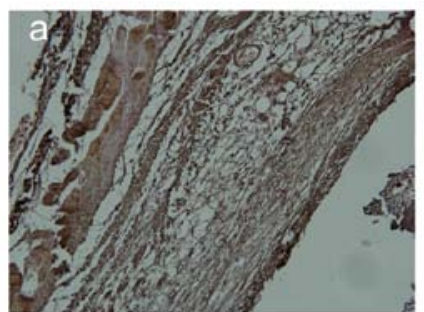

control

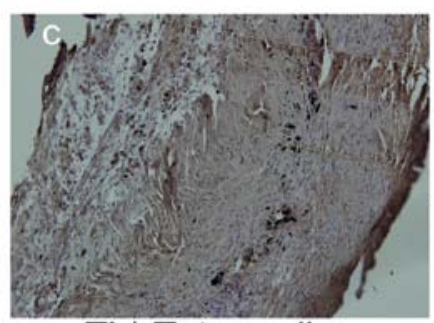

$\mathrm{Ti}+$ Tetracycline

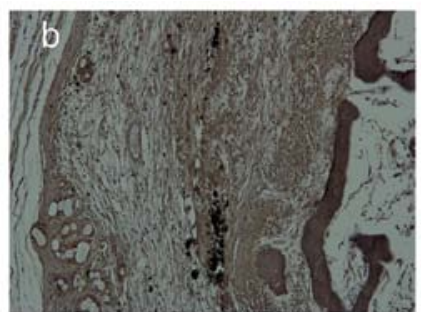

$\mathrm{Ti}$

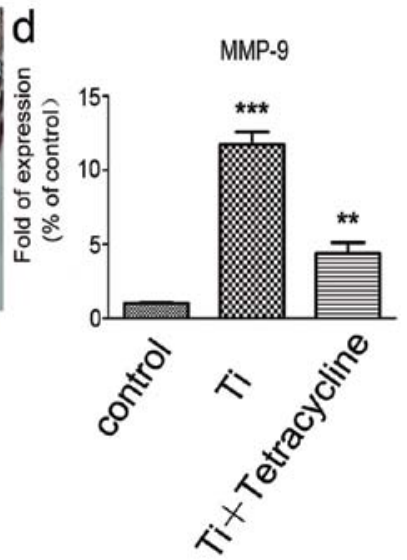

Figure 3. Immunohistochemical detection of MMP-9 in the murine osteolysis model. Air pouch in the (a) control, (b) Ti and (c) Ti plus tetracycline group (magnification, x200). (d) Real-time RT-PCR analysis of the MMP-9 expression in pouch tissues. ${ }^{* *} \mathrm{P}<0.05,{ }^{* * *} \mathrm{P}<0.05$ compared to the control group.

Real-time RT-PCR assay indicated that MMP-9 gene transcription was significantly higher in the Ti plus tetracycline group compared to the control group $(\mathrm{P}<0.001)$ but was less marked compared to the Ti group. There was also a significant difference in the gene transcripts of MMP-9 between the Ti and Ti plus tetracycline groups (Fig. 3d). Real-time RT-PCR analysis also demonstrated that $\mathrm{Ti}$ particles stimulated a significant increase in the gene expression of inflammatory mediators in air pouch tissues, while tetracycline treatment significantly attenuated these responses.

Effect of tetracycline on TNF- $\alpha$ expression. Staining for TNF- $\alpha$ in the Ti group was significantly stronger compared to the control group (Fig. $4 \mathrm{a}-\mathrm{c}$ ). In the Ti plus tetracycline
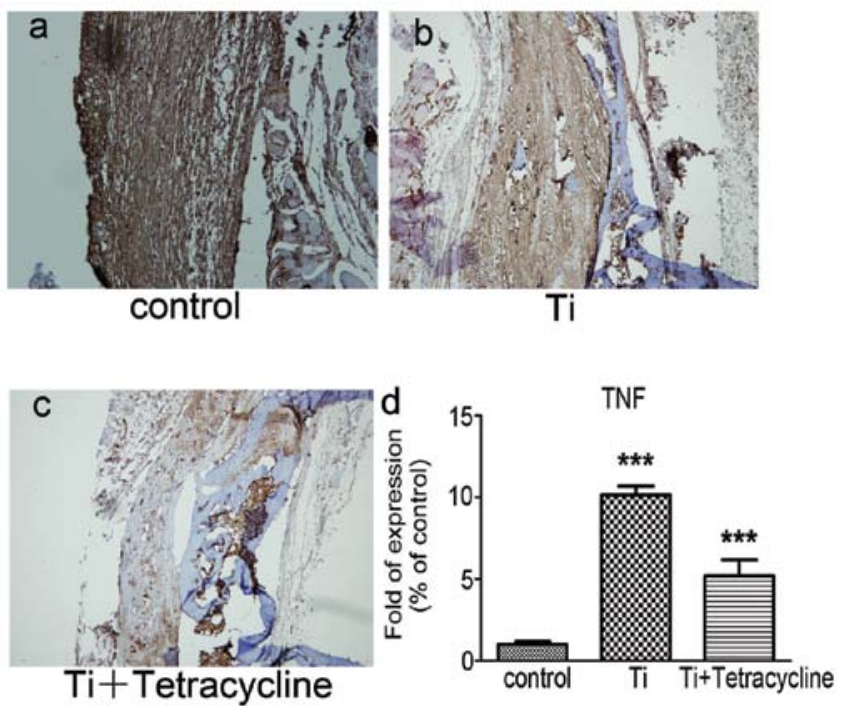

Figure 4. Immunohistochemical detection of TNF- $\alpha$ in the murine osteolysis model. Air pouch in the (a) control, (b) Ti and (c) Ti plus tetracycline group (magnification, x200). (d) Real-time RT-PCR analysis of TNF- $\alpha$ expression in pouch tissues. ${ }^{* * * *} \mathrm{P}<0.05$ compared to the control group.

group there was evidence of significant suppression of the $\mathrm{Ti}$ particle-induced increase in TNF- $\alpha(\mathrm{P}<0.05)$.

The gene levels of TNF- $\alpha$ determined by real-time RT PCR are displayed in Fig. 4d. The Ti particles significantly increased TNF- $\alpha$ gene expression compared to the control group $(\mathrm{P}<0.001)$. In addition, the number of gene copies of TNF- $\alpha$ was significantly reduced in the Ti plus tetracycline group $(\mathrm{P}<0.001)$.

Effect of tetracycline on RANK and RANKL expression. Immunohistochemical staining, as depicted in Figs. 5a-c and 6a-c, demonstrated increases in staining for both RANK and RANKL in the Ti group compared to the control group. RANKL staining was discovered at the interface between the pouch membranes and the implanted bone tissue and was significantly decreased in the tetracyclinetreated group $(\mathrm{P}<0.05)$.

Real-time RT-PCR demonstrated that the Ti particles significantly $(\mathrm{P}<0.05)$ increased the expression of both RANK and RANKL gene transcripts in comparison with the control 


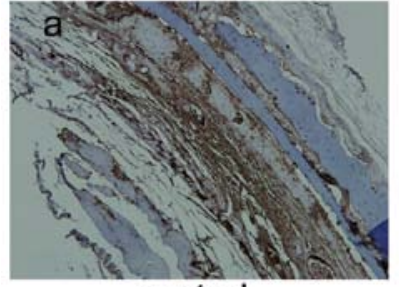

control

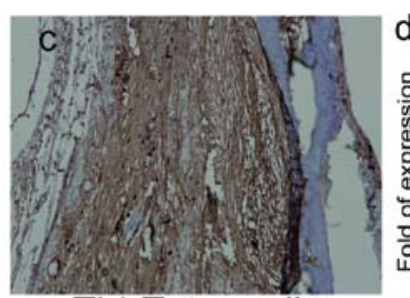

$\mathrm{Ti}+$ Tetracycline

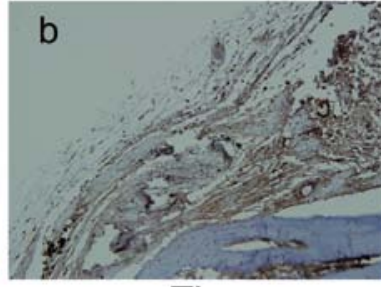

$\mathrm{Ti}$

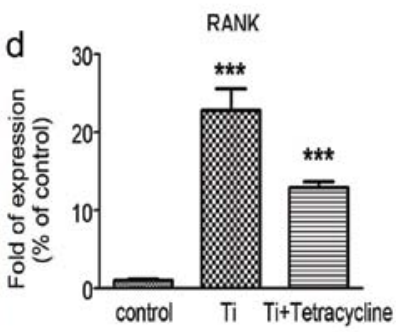

Figure 5. Immunohistochemical detection of RANK in the murine osteolysis model. Air pouch in the (a) control, (b) Ti and (c) Ti plus tetracycline group (magnification, x200), (d) Real-time RT-PCR analysis of the RANK expression in pouch tissues. ${ }^{* * *} \mathrm{P}<0.05$ compared to the control group.

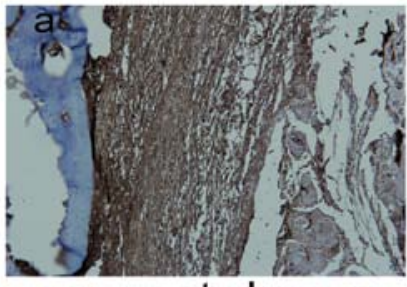

control

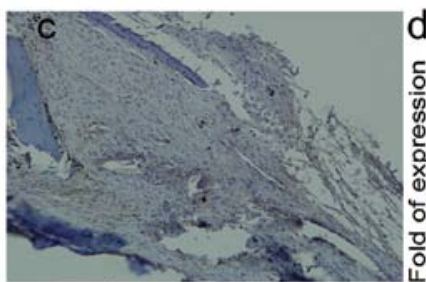

$\mathrm{Ti}+$ Tetracycline

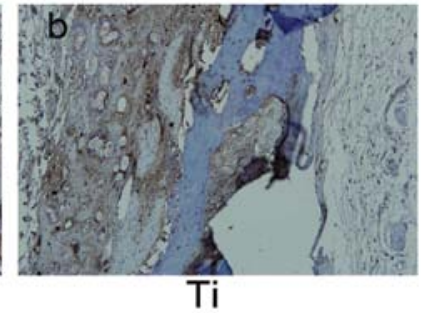

$\mathrm{Ti}$

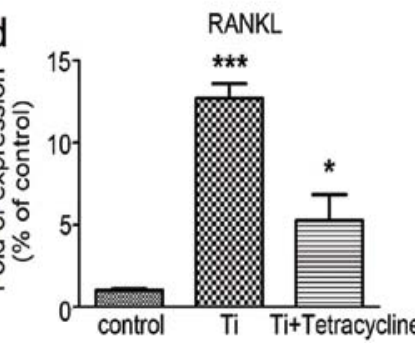

Figure 6. Immunohistochemical detection of RANKL in the murine osteolysis model. Air pouch in the (a) control, (b) Ti and (c) Ti plus tetracycline group (magnification, x200). (d) Real-time RT-PCR analysis of the RANKL expression in pouch tissues. ${ }^{*} \mathrm{P}<0.05,{ }^{* * *} \mathrm{P}<0.05$ compared to the control group.

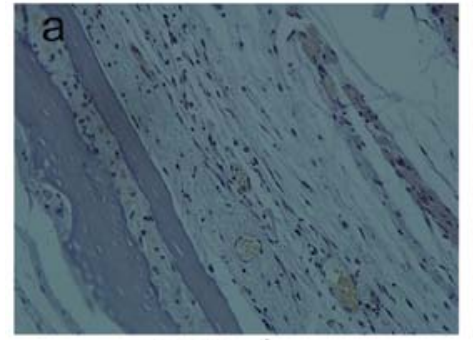

control

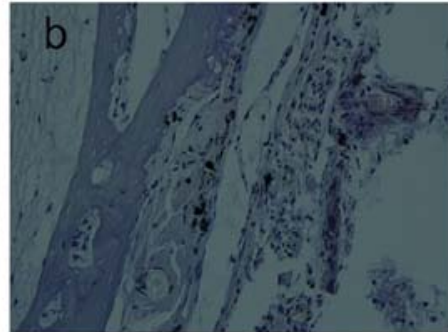

$\mathrm{Ti}$

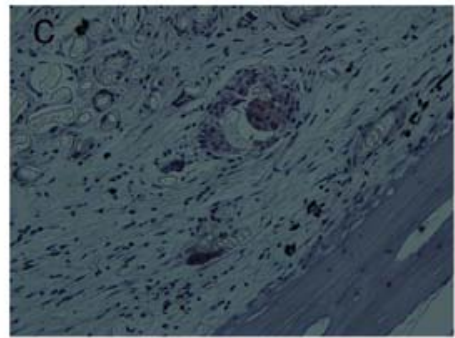

Ti+Tetracycline

Figure 7. Representative TRAP stain in paraffin tissues. TRAP-positive cells were stained dark red in the pouch membranes. (a) Control, (b) Ti and (c) Ti plus tetracycline groups.

group (Figs. 5d and 6d). Exposure to tetracycline significantly reduced $(\mathrm{P}<0.001)$ the increases in RANK and RANKL gene transcripts compared with the Ti group.

Effect of tetracycline on Ti-particle-induced osteoclastogenesis. Paraffin sectioned calvaria-implanted pouches were stained to identify osteoclast-like cells. A typical TRAPstained photomicrograph exhibiting an accumulation of dark purple TRAP-positive cells at the interface between the implanted calvaria and the pouch membrane is displayed in Fig. 7. Relatively few TRAP-positive cells were present in the control or Ti plus tetracycline groups.

\section{Discussion}

Wear debris-induced osteolysis and aseptic loosening is the most common complication of total joint replacement surgery (2). During the 10-year period following joint replacement, up to $20 \%$ of patients experience radiographic loosening of the joint (24). From the data available to date, persistent debris-induced inflammation and osteoclastogenesis may be critical factors involved in the etiology of aseptic implant loosening $(25,26)$. Failed implants are often surrounded by a synovial membrane-like interface, known as the interfacial membrane, which plays a significant role in tissue destruction and causes characteristic chronic inflammation in the periimplant area $(27,28)$. The wear debris stimulus stimulates the gathering of fibroblasts, macrophages, and foreign body giant cells in interfacial membranes where they produce multiple proinflammatory cytokines such as TNF- $\alpha$ and IL-6 $(29,30)$. In addition, the abundance of MMPs produced by these cells facilitates bone resorption around implants resulting in aseptic loosening (31).

MMP-9 is produced by multinucleated giant cells in response to wear debris at the interface membranes where it may facilitate orthoclastic bone resorption by degradation of extracellular matrix macromolecules present around and on the surface of the bone trabeculae (32). Previous studies have demonstrated that receptor activator of NF- $\mathrm{B}$ (RANK) and RANK ligand (RANKL) are key factors that regulate bone turnover and are present in tissues near the site of periprosthetic osteolysis (18). However, whether MMP-9 acts as 
an upstream regulator of inflammation and bone resorptioninduced RANK/RANKL signaling during inflammatory osteoclastogenesis remains ambiguous.

The present study was undertaken in a murine osteolysis model to examine the hypothesis that inhibition of Ti particle-induced inflammatory osteoclastogenesis by treatment with an MMP-9 inhibitor occurs through the downregulation of RANK/RANKL, MMP-9 and TNF- $\alpha$. Immunohistochemistry and real-time RT-PCR for proinflammatory cytokines demonstrated that bone implantation and Ti particle stimulation of air pouches significantly increased protein and gene expression of MMP-9 and TNF- $\alpha$, resulting in a significant inflammatory reaction (33-35). We also demonstrated that TNF- $\alpha$ is produced in response to wear particles and that it affects downstream processes resulting in osteolysis $(24,36)$. We used tetracycline, as a MMP-9 inhibitor on the basis that it has been observed to have a role in the treatment of several ophthalmologic diseases by affecting the expression of MMP-9 and TNF- $\alpha$ (37). Additionally, the tetracycline derivative, doxycycline, reduces MMP-9-mediated inflammation and the downstream effects of inflammation $(16,38,39)$. This provides a potentially important therapeutic strategy for the management of debris-induced inflammation.

In our study, real-time RT-PCR displayed that the number of gene copies of MMP-9 was significantly reduced by exposure to tetracycline. In addition, immunohistochemical studies suggested that tetracycline impeded MMP-9 expression in Ti particle pouches. These findings suggest that tetracycline suppresses the activation and expression of MMP-9 in Ti particle-induced inflammatory osteolysis.

Data from our H\&E-stained sections revealed that the presence of Ti particles increased cellular infiltration and pouch membrane thickness by stimulating tissue inflammation. However, membrane thickness and the frequency of cell infiltration were both significantly reduced in animals treated with tetracycline. In addition, we were able to identify TRAP-positive osteoclasts, by TRAP staining and discovered that tetracycline reduced the number of TRAP-positive cells in Ti particle-induced pouches. These findings suggest that tetracycline has the potential to decrease bone resorption in the murine air pouch osteolysis model.

The expression of both RANK and RANKL genes in our mouse model of peri-prosthesis tissue was well defined. Other studies have demonstrated that Ti particles significantly increased RANK/RANKL gene expression in murine pouch tissues $(17,40)$ and it has been proposed that activation of the RANK/RANKL pathway may play a prominent role in the bone loss associated with aseptic loosening (40). In our study, tetracycline, an MMP-9 inhibitor, significantly reduced the expression of RANK and RANKL. MMP-9 has numerous effects on osteoclast and osteoblast function (41-43), and previous research has demonstrated that wear debris increases the expression of MMP-9 and TNF- $\alpha$, both in vivo and in vitro (44). These findings are consistent with our results.

TNF- $\alpha$ is considered to stimulate osteoclastogenesis through the upregulation of RANKL production in osteoblasts and through the stimulation of RANK expression in osteoclasts (45). This is in accordance with the observation that RANK and RANKL are downstream signaling molecules of the MMP-9 pathway. In our study, Ti particles increased the gene expression of RANK and RANKL in comparison with the control group, and tetracycline significantly decreased the expression of RANK and RANKL and reduced the number of TRAP-positive cells, compared with the control group. These findings suggest that tetracycline suppresses the expression of the RANK and RANKL signaling pathways in the presence of $\mathrm{Ti}$ particle-induced inflammatory osteolysis. However, a previous study demonstrated that RANKL signals through TRAF6 and that NFATc1 is a downstream effector of RANKL signaling to modulate MMP-9 gene expression during osteoclast differentiation (46). There may, therefore, be an interrelationship between MMP-9 and RANK/RANKL signaling in the development of inflammatory osteolysis, suggested by the fact that treatment with an MMP-9 inhibitor significantly reduced the levels of RANK/RANKL gene expression in the current study.

We used a murine model in this study, as it provides a sensitive and cost-effective way to investigate the responses of particle-induced inflammatory osteolysis in aseptic loosening and offers advantages over previously reported models (47). We used air pouches implanted with genetically identical donor mouse calvarial bone to gain insight into changes in bone resorption and to provide a model of chronic inflammation observed in inflammatory osteolysis. Collectively, our results of the murine osteolysis model suggest that MMP-9 plays a critical role in the process of wear debris-induced inflammatory osteolysis. MMP-9 inhibition by tetracycline impeded the expression of RANK/RANKL in the development of Ti-particle-induced inflammatory osteolysis, indicating that MMP-9 inhibition is a potential therapeutic target for the prevention of wear debris-induced inflammatory osteolysis. The optimal clinical protocol for tetracycline in this type of inflammation has yet to be determined through further research.

\section{Acknowledgements}

This study was supported by the Interdisciplinary (Engineering-Medical) Research Fund of Shanghai Jiao Tong University (grant no. YG2011MS30), the Shanghai Municipal Health Bureau Science Fund for Young Scholars (grant no. 2010QJ036A) and the National Natural Science Foundation of China (grant no. 81171688).

\section{References}

1. Horowitz SM, Doty SB, Lane JM and Burstein AH: Studies of the mechanism by which the mechanical failure of polymethylmethacrylate leads to bone resorption. J Bone Joint Surg Am 75: 802-813, 1993.

2. Harris WH: Wear and periprosthetic osteolysis: the problem. Clin Orthop Relat Res: 66-70, 2001.

3. Ingham $E$ and Fisher J: The role of macrophages in osteolysis of total joint replacement. Biomaterials 26: 1271-1286, 2005.

4. Holt G, Murnaghan C, Reilly J and Meek RM: The biology of aseptic osteolysis. Clin Orthop Relat Res 460: 240-252, 2007.

5. von Knoch F, Wedemeyer C, Heckelei A, et al: Promotion of bone formation by simvastatin in polyethylene particle-induced osteolysis. Biomaterials 26: 5783-5789, 2005.

6. Peng X, Tao K, Cheng T, Zhu J and Zhang X: Efficient inhibition of wear debris-induced inflammation by locally delivered siRNA. Biochem Biophys Res Commun 377: 532-537, 2008.

7. Takagi M, Konttinen YT, Santavirta S, et al: Extracellular matrix metalloproteinases around loose total hip prostheses. Acta Orthop Scand 65: 281-286, 1994. 
8. Nawrocki B, Polette M, Burlet $\mathrm{H}$, Birembaut $\mathrm{P}$ and Adnet JJ: Expression of gelatinase A and its activator MT1-MMP in the inflammatory periprosthetic response to polyethylene. J Bone Miner Res 14: 288-294, 1999.

9. Takei I, Takagi M, Santavirta S, et al: Matrix metalloproteinases and tissue inhibitors of metalloproteinases in joint fluid of the patients with loose artificial hip joints. J Biomed Mater Res 45: 175-183, 1999.

10. Wagner S, Gollwitzer H, Wernicke D, Langer R, Siebenrock KA and Hofstetter W: Interface membrane fibroblasts around aseptically loosened endoprostheses express MMP-13. J Orthop Res 26: 143-152, 2008

11. Reponen P, Sahlberg C, Munaut C, Thesleff I and Tryggvason K: High expression of $92-\mathrm{kD}$ type IV collagenase (gelatinase B) in the osteoclast lineage during mouse development. J Cell Biol 124: 1091-1102, 1994.

12. Tsai CH, Chen YJ, Huang FM, Su YF and Chang YC: The upregulation of matrix metalloproteinase-9 in inflamed human dental pulps. J Endod 31: 860-862, 2005.

13. Wucherpfennig AL, Li YP, Stetler-Stevenson WG, Rosenberg AE and Stashenko P: Expression of $92 \mathrm{kD}$ type IV collagenase/gelatinase B in human osteoclasts. J Bone Miner Res 9: 549-556, 1994

14. De Jong PT, Tigchelaar W, Van Noorden CJ and Van der Vis HM: Polyethylene wear particles do not induce inflammation or gelatinase (MMP-2 and MMP-9) activity in fibrous tissue interfaces of loosening total hip arthroplasties. Acta Histochem 113: 556-563, 2011.

15. Zhang C, Tang TT, Ren WP, Zhang XL and Dai KR: Inhibiting wear particle-induced osteolysis with doxycycline. Acta Pharmacol Sin 28: 1603-1610, 2007.

16. Gu Y, Lee HM, Sorsa T, Simon SR and Golub LM: Doxycycline inhibits mononuclear cell-mediated connective tissue breakdown. FEMS Immunol Med Microbiol 58: 218-225, 2010.

17. Geng D, Xu Y, Yang H, et al: Protection against titanium particle induced osteolysis by cannabinoid receptor 2 selective antagonist. Biomaterials 31: 1996-2000, 2010.

18. Holding CA, Findlay DM, Stamenkov R, et al: The correlation of RANK, RANKL and TNFalpha expression with bone loss volume and polyethylene wear debris around hip implants Biomaterials 27: 5212-5219, 2006.

19. Wedemeyer C, Neuerburg C, Pfeiffer A, et al: Polyethylene particle-induced bone resorption in alpha-calcitonin generelated peptide-deficient mice. J Bone Miner Res 22: 1011-1019, 2007.

20. Yang SY, Wu B, Mayton L, et al: Protective effects of IL-1Ra or vIL-10 gene transfer on a murine model of wear debris-induced osteolysis. Gene Ther 11: 483-491, 2004.

21. Ren W, Yang SY and Wooley PH: A novel murine model of orthopaedic wear-debris associated osteolysis. Scand J Rheumatol 33: 349-357, 2004

22. Mao X, Pan X, Zhao S, Peng X, Cheng T and Zhang X: Protection against titanium particle-induced inflammatory osteolysis by the proteasome inhibitor bortezomib in vivo. Inflammation 35: 1378-1391, 2012.

23. Wooley PH, Morren R, Andary J, et al: Inflammatory responses to orthopaedic biomaterials in the murine air pouch. Biomaterials 23: 517-526, 2002

24. Sams JD, Milbrandt JC, Froelich JM, Rainville AD and Allan DG: Hospital outcome after emergent vs elective revision total hip arthroplasty. J Arthroplasty 25: 826-828, 2010.

25. Epstein NJ, Warme BA, Spanogle J, et al: Interleukin-1 modulates periprosthetic tissue formation in an intramedullary model of particle-induced inflammation. J Orthop Res 23 501-510, 2005

26. Waddell J, Pritzker KP and Boynton EL: Increased cytokine secretion in patients with failed implants compared with patients with primary implants. Clin Orthop Relat Res 434: $170-176,2005$

27. Atkins RM, Langkamer VG, Perry MJ, Elson CJ and Collins CM: Bone-membrane interface in aseptic loosening of total joint arthroplasties. J Arthroplasty 12: 461-464, 1997.

28. Goodman SB, Huie P, Song Y, et al: Cellular profile and cytokine production at prosthetic interfaces. Study of tissues retrieved from revised hip and knee replacements. J Bone Joint Surg Br 80: 531-539, 1998.
29. Goldring SR, Schiller AL, Roelke M, Rourke CM, O'Neil DA and Harris WH: The synovial-like membrane at the bonecement interface in loose total hip replacements and its proposed role in bone lysis. J Bone Joint Surg Am 65: 575-584, 1983.

30. Kim KJ, Rubash HE, Wilson SC, D'Antonio JA and McClain EJ: A histologic and biochemical comparison of the interface tissues in cementless and cemented hip prostheses. Clin Orthop Relat Res 287: 142-152, 1993.

31. Ma GF, Ali A, Verzijl N, et al: Increased collagen degradation around loosened total hip replacement implants. Arthritis Rheum 54: 2928-2933, 2006.

32. Yokohama Y, Matsumoto T, Hirakawa M, et al: Production of matrix metalloproteinases at the bone-implant interface in loose total hip replacements. Lab Invest 73: 899-911, 1995.

33. Velard F, Laurent-Maquin D, Braux J, et al: The effect of zinc on hydroxyapatite-mediated activation of human polymorphonuclear neutrophils and bone implant-associated acute inflammation. Biomaterials 31: 2001-2009, 2010.

34. Takagi M, Tamaki Y, Hasegawa H, et al: Toll-like receptors in the interface membrane around loosening total hip replacement implants. J Biomed Mater Res A 81: 1017-1026, 2007.

35. Laquerriere $\mathrm{P}$, Grandjean-Laquerriere A, Addadi-Rebbah $\mathrm{S}$, et al: MMP-2, MMP-9 and their inhibitors TIMP-2 and TIMP-1 production by human monocytes in vitro in the presence of different forms of hydroxyapatite particles. Biomaterials 25: 2515-2524, 2004

36. Taki N, Tatro JM, Lowe R, Goldberg VM and Greenfield EM: Comparison of the roles of IL-1, IL-6, and TNFalpha in cell culture and murine models of aseptic loosening. Bone 40: 1276-1283, 2007.

37. Federici TJ: The non-antibiotic properties of tetracyclines: clinical potential in ophthalmic disease. Pharmacol Res 64: 614-623, 2011.

38. Lau AC, Duong TT, Ito S, Wilson GJ and Yeung RS: Inhibition of matrix metalloproteinase-9 activity improves coronary outcome in an animal model of Kawasaki disease. Clin Exp Immunol 157: 300-309, 2009.

39. Samtani S, Amaral J, Campos MM, Fariss RN and Becerra SP: Doxycycline-mediated inhibition of choroidal neovascularization. Invest Ophthalmol Vis Sci 50: 5098-5106, 2009.

40. Ren WP, Markel DC, Zhang R, et al: Association between UHMWPE particle-induced inflammatory osteoclastogenesis and expression of RANKL, VEGF, and Flt-1 in vivo. Biomaterials 27: 5161-5169, 2006.

41. Hyun JJ, Chun HJ, Keum B, et al: Effect of omeprazole on the expression of transcription factors in osteoclasts and osteoblasts. Int J Mol Med 26: 877-883, 2010.

42. Sriarj W, Aoki K, Ohya K, Takagi Y and Shimokawa H: Bovine dentine organic matrix down-regulates osteoclast activity. J Bone Miner Metab 27: 315-323, 2009.

43. Tat SK, Pelletier JP, Mineau F, Caron J and Martel-Pelletier J: Strontium ranelate inhibits key factors affecting bone remodeling in human osteoarthritic subchondral bone osteoblasts. Bone 49: 559-567, 2011.

44. Choi MG, Koh HS, Kluess D, et al: Effects of titanium particle size on osteoblast functions in vitro and in vivo. Proc Natl Acad Sci USA 102: 4578-4583, 2005.

45. Jurado S, Garcia-Giralt N, Diez-Perez A, et al: Effect of IL-1beta, PGE(2), and TGF-betal on the expression of OPG and RANKL in normal and osteoporotic primary human osteoblasts. J Cell Biochem 110: 304-310, 2010.

46. Sundaram K, Nishimura R, Senn J, Youssef RF, London SD and Reddy SV: RANK ligand signaling modulates the matrix metalloproteinase-9 gene expression during osteoclast differentiation. Exp Cell Res 313: 168-178, 2007.

47. Mao X, Pan X, Cheng T and Zhang X: Therapeutic potential of the proteasome inhibitor bortezomib on titanium particleinduced inflammation in a murine model. Inflammation 35: 905-912, 2012. 